\title{
The effects of AO systems on polarized light
}

\author{
M. Stangalini*a ${ }^{\text {L. Giovannelli }}{ }^{\mathrm{b}}$, D. Del Moro ${ }^{\mathrm{b}}$ and F. Berrilli ${ }^{\mathrm{b}}$ \\ ${ }^{a}$ INAF-OAR Osservatorio Astronomico di Roma, Monte Porzio Catone, Italy \\ ${ }^{b}$ Dept. of Physics, University of Rome Tor Vergata, Rome, Italy
}

\begin{abstract}
Spectropolarimetry is nowadays one of the most used tool to investigate small scale $(100 \mathrm{~km})$ magnetic fields in the Sun's atmosphere. In addition, theforthcoming 4-meter class solar telescopes will provide an unprecedented view of the solar magnetism with an accuracy $\left(10^{-4}\right)$ never reached before, and on spatial scales which are at least twice as smaller. For this reason MCAO systems providing high Strehl ratios on a large field of view are being developed. Thus, the study of any possible effect of such AO systems on the polarization accuracy has to be carefully assessed. In this contribution we present preliminary results of laboratory tests conducted with the aim of evaluating possible drawbacks of the use of deformable mirrors on the spectropolarimetric accuracy.
\end{abstract}

Keywords: Spectropolarimetry, Solar instrumentation, Adaptive optics

\section{INTRODUCTION}

Polarization measurements have quickly become a standard tool, in recent years, in solar physics research for the investigation of the magnetic fields in the Sun's atmosphere ${ }^{1,2,3}$, and they are becoming an increasingly helpful tool also in nighttime astronomy ${ }^{4}$, where they have been employed to study stellar magnetic fields, accretion disks, and in the detection exoplanets ${ }^{5,6}$. However, the spatial resolution required to achieve the main scientific goals is high, and one needs to employ complex AO (Adaptive Optics) systems to reach these goals. At the same time, also a very high polarimetric sensitivity is required (e.g. $10^{-6}$ to detect mall magnetic structures $20-30 \mathrm{~km}$ large in the solar photosphere).

It is a well-known result the polarization degree of the light depends on the angle of incidence on a steady mirror surface. The transmission coefficients of light with polarization planes parallel or perpendicular to the reflection plane are dependent on the angle of incidence, as one can see from the Fresnel equations. This effect is already taken into account in the calibration of presently used spectropolarimeters, but little is known about the effect of deformable mirrors on the polarization of light in high precision spectropolarimetric measurements. These effects are, in fact, currently assumed negligible, within the spectropolarimetric accuracy limits. However, has aforementioned, next four-meter class solar telescopes like $\mathrm{EST}^{7,8}$ (European Solar Telescope) and DKIST ${ }^{9,10}$ (The Daniel K. Inouye Solar Telescope, formerly the Advanced Technology Solar Telescope, ATST) will incorporate sophisticated MCAO (Multi-Conjugated AO) systems in order to increase the corrected field-of-view. These systems will be made of two or more deformable mirrors. On the other hand, these telescopes will also require a polarimetric sensitivity of $10^{-6}$ which has never reached before. In this context, it is recommended to study any possible effects introduced by non-steady mirror surfaces on the polarization.

A deformable mirror can be in fact seen as a set of small steady mirrors that locally can alter the polarization state of light. In addition, although the local slope of the deformable mirror is generally really small compared to the angle of incidence on folding mirrors along the optical path, deformable mirrors are not steady and therefore they may introduce a time-dependent effect on the polarization which would be difficult to calibrate. The effects of AO on polarization have been already studied through numerical simulations in the solar case ${ }^{11}$.

Motivated by that, in this contribution we show preliminary results obtained from the measurements of polarization effects of a deformable mirror (DM) in a very simple optical setup. In particular, we studied the effect of a DM in both open loop and closed loop operations to simulate conditions typical of astronomical observations. 


\section{OPTICAL SETUP AND MEASUREMENTS}

\subsection{Optical setup}

The optical setup used in our experiment consists of a 48 elements membrane DM and two linear polarizers (see Fig.1). An incoming laser beam $(630 \mathrm{~nm})$ passes through the first linear polarizer before being reflected by the DM and sent to second linear polarizer which is rotated to obtain the maximum transmission of the incoming light when the DM is flat. After the second linear polarizer, a beam splitter divides the beam into two parts; one is sent to a low-order ShackHartmann wave-front sensor (WFS), the other on a CCD sensor which is conjugated to the pupil plane. In this condition the beam is not focused but the CCD sensor images the pupil plane. The deformations of the DM that change the polarization of the beam and therefore reduce the signal onto the detector. We chose this configuration to maximize the signal and reduce the photon noise. The integration time is set to $0.1 \mathrm{~s}$ and the cadence is $1 \mathrm{~s}$.

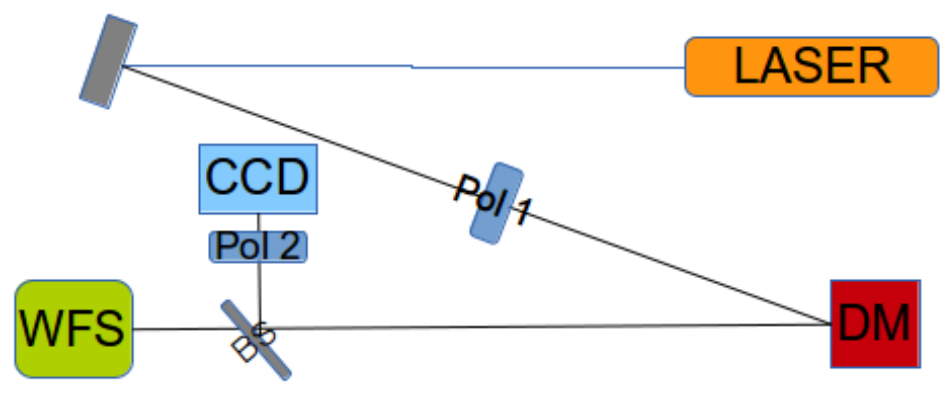

Fig. 1. Optical layout of the experiment. A collimated laser beam goes through a linear polarizer and then on a 37 elements DM. After the DM the beam encounters a second linear polarizer before reaching a beam splitter and the CCD and the Shack-Hartmann wave-front sensor. The collimation optics is omitted in the scheme for the sake of simplicity.

\subsection{Methods}

As mentioned above, our main goal is to estimate the effect of the DM deformation on the polarized light into two conditions: open loop and closed loop. The general strategy is to measure polarization effects as variation of light intensity with respect to the reference (flat mirror) case. Any depolarization induced by the local curvature of the DM, will reflect in a change of the amount of light transmitted by the second linear polarizer.

The reference measure has been acquired with the DM reproducing a flat target shape. Then we performed two other intensity measure: one in a open loop condition, the second in a closed loop condition. For both the reference and the open loop condition, the shape of the DM is frozen after few seconds of closed loop operation, to allow the DM to reach the target shape down to a minimum residual shape error. The accuracy of the shape is measured by the WFS and the residual RMS error from the flat shape is 0.03 waves.

In the open loop condition, the DM is kept steady during the measurements with a fixed shape. To do this, a defocus aberration is sent to the mirror and the resultant shape is checked by WFS. After that, the mirror shape is frozen while the CCD acquires a series of images ( 200 images). 
In closed loop operations, a target shape is sent to the DM and it works in closed loop conditions. This means that the shape of the DM is monitored by the WFS and corrections are sent continuously to minimize the residual errors from the target shape. This configuration mimics the ones employed during astronomical observations.

In Tab. 1 we report the list of target shapes used during our measurements and their quantity and RMS error in terms of standard Zernike modal coefficients ${ }^{12}$. We recall that $\mathrm{Z} 4$ corresponds to a pure defocus aberration.

Table 1. List of DM shapes and their relative residual error with respect the target.

\begin{tabular}{|llll|}
\hline \multicolumn{4}{c|}{ DM shapes } \\
Open loop & \multicolumn{3}{c|}{ Closed loop } \\
\hline $0.3 Z 4$ & RMS error 0.07 waves & $0.4 Z 4$ & RMS error 0.06 waves \\
Reference & RMS error 0.03 waves & & \\
\hline Flat: & R & & \\
\hline
\end{tabular}

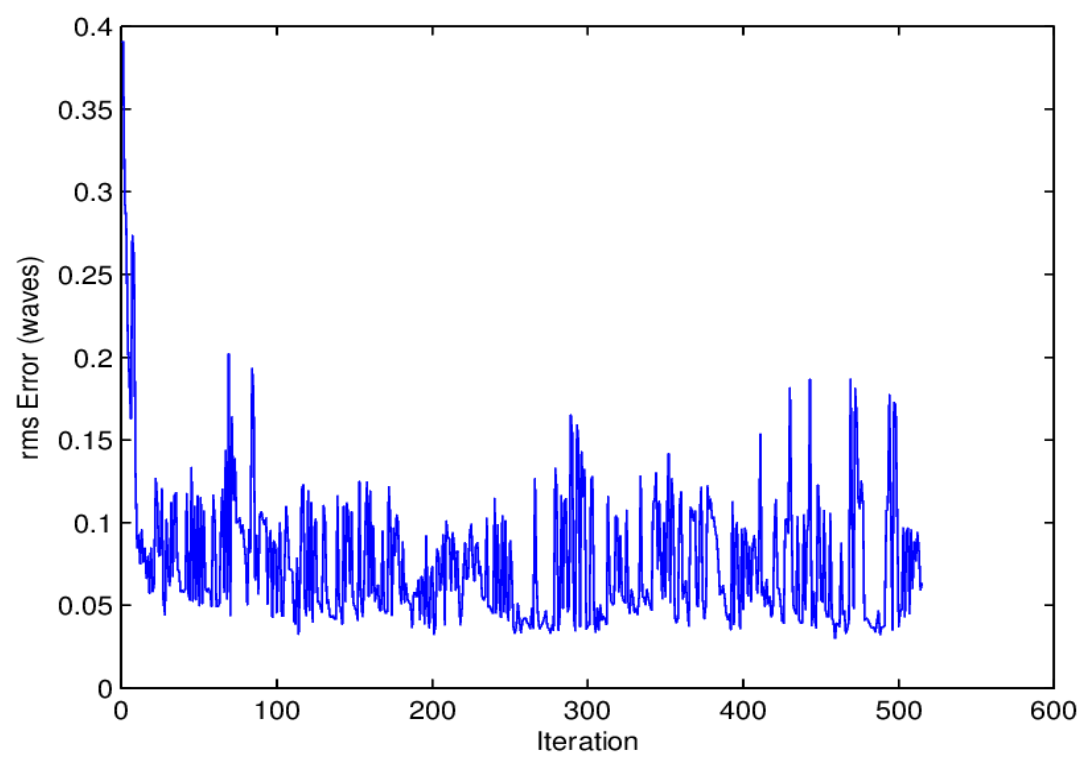

Fig.2. Residual oscillations of the RMS error during the closed loop.

In Fig. 2 we show the residual RMS error in the closed loop condition. After the first steps, the loop is closed and the residual error, with respect to the target, is minimized. From this moment on, the CCD starts acquiring images. The RMS error fluctuates with an amplitude of $\sim 0.05$ waves during the closed loop iterations.

\section{RESULTS}

After registering all the images of the closed loop and open loop sequences with sub-pixel accuracy using an FFT-based technique, we selected a region of interest (ROI) 500x500 pixels large and centered on the images. We then computed 
the total intensity in this region as a function of time. After removing a low order trend from each sequence of images (open and closed loop) due to a variation in the incoming laser light, we divided the recorded intensity values by the average intensity of the reference run in order to estimate the relative intensity decrease with respect to the flat mirror condition. The results of this analysis are shown in figure 3.

Interestingly, we measure a decrease of the average intensity with respect to the reference both in open and closed loop conditions. This effect correlates with the amount of the $\mathrm{Z} 4$ coefficient value: it is $\sim 1 \%$ for a 0.3 waves defocus and $2.5 \%$ for a $0.4 \%$ defocus.

Moreover, in the closed loop condition, the signal shows fluctuation much higher than those of the reference around the relative average value. If we take the reference signal fluctuation as due to photon noise fluctuation, we can hypothesize that the larger effect on the closed loop signal is mainly induced by to the variation of the shape of the DM, due to the continuous corrections of the AO system. Such oscillations are of the order of $1 \%$ of the incoming intensity.

It is worth noting that the reduction of the average intensity associated to the mirror deformation may be also explained by an increase of the amount of stray light as an effect of the defocus induced by the DM itself. Part of the photons can be in fact spread outside the ROI reducing the signal. If this effect can explain the reduction of the average signal value, it can hardly explain the strong fluctuations during the closed loop operation. In the latter case, the shape of the DM is varying very little with respect to its nominal shape (RMS $\sim 0.05$ waves) and we do not expect the shape of the beam incoming onto the CCD to vary significantly.

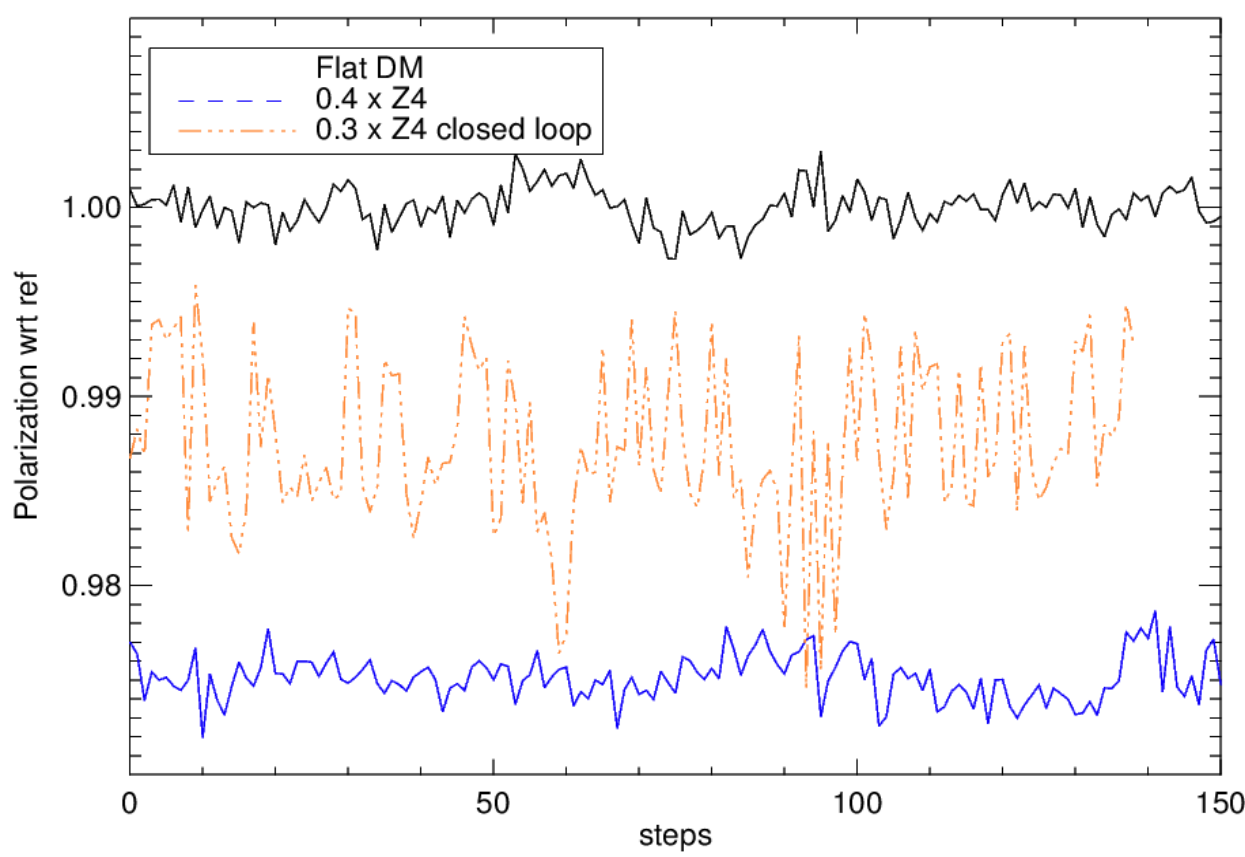

Fig. 3 Relative amount of total linear polarization for the closed loop and the open loop, with respect to the reference (flat mirror) as a function of time.

\section{DISCUSSIONS AND CONCLUSIONS}

In this work we have shown preliminary results of the investigation of the effects of a DM on the polarized light. These effects are commonly assumed negligible in both nighttime and solar astronomy. 
Our results show the presence of an intensity change in the polarized light up to a degree of $\sim 1 \%$. This change is obtained by introducing a very low order aberration on the DM (defocus). In addition, when the DM operates in closed loop with the WFS, a modulation with an amplitude of the same order is also seen.

In any case, results must be interpreted with care since the measured change in intensity can be due either to the depolarization of the beam, of to an intensity modulation due to stray light effects.

In the second case, part of the photons in the optical beam may be dispersed outside the main collimated beam due to the aberrations introduced by the deformable mirror. In this case, it is difficult to assess the amount of photon losses due to stray light. However, while the increasing reduction of the emergent intensity for increasing defocus amounts can be attributed to this effect in open loop conditions, it is worth stressing that in closed loop operations the deformable mirror is subject to very small relative fluctuations of its shape with respect to the target. These small oscillations from the target shape are much smaller ( 0.05 waves) than the amplitude of defocus which are applied in the different runs of our experiment (0.3-0.4 waves with respect to flat mirror). Despite this, the closed loop experiment produces oscillations of the emergent polarized intensity with an amplitude of the same order as the reduction of intensity with respect to the flat mirror, in the open loop conditions. For this reason, and since the shape of the DM is subject only to tiny deformations in closed loop, which reflect in a small amount of stray light, it is reasonable to think that a large part of this oscillation is actually due to a depolarization of the optical beam.

In any case, further investigation of this effect must be carried on. In particular, more experimental tests and calibrations aimed at disentangling the two possible effects must be performed.

However, independently of this, the $1 \%$ amplitude oscillation sets up an upper limit for optical beam depolarization. In the case a variation of the order of $1 \%$ is totally due to polarization, this would imply that a non-negligible effect is to be expected during the acquisition of science data. This in turn requires a specific strategy to minimize this effect. Differently from the standard spectropolarimetric calibrations usually adopted, which makes use of static Mueller matrices to correct for the effects of the optical components along the path, the DM induced effect requires a more sophisticated strategy with a strongly time-dependent calibration. To this regard, it would be crucial to record the DM deformation during the closed loop operation in order to properly take into account this effect and perform a post-facto calibration. Unfortunately, this implies a large amount of data to be collected and stored.

On the other hand, intrinsic intensity oscillations can also dramatically affect the spectropolarimetric measurements by altering Stokes profiles which are obtained by scanning in wavelength magnetic sensitive spectral lines, as in FabryPerot 2D interferometers. In the case of a spectropolarimetric measurement of the solar atmosphere, in fact, a scan in wavelength can take several seconds to complete. If the intensity changes due to the DM are on time scales smaller than the scanning time, the intensity changes can alter the spectropolarimetric profiles, unless those changes are much more rapid than the integration time at each spectral wavelength during the spectral scan. In this latter case, in fact, if the seeing is stable enough, the effect of averaging over several oscillations produced by the DM would compensate.

\section{REFERENCES}

1. E. L. Degl'Innocenti, “Recipes for Solar Polarimetry,” Solar Surface Magnetism, pp. 29-35, 1994.

2. J. O. Stenflo, "Polarized Radiation Diagnostics of Solar Magnetic Fields," Astrophysical Spectropolarimetry, pp. 55-100, 2001.

3. J. C. del Toro Iniesta, "Solar polarimetry," Introduction to Spectropolarimetry, pp. 64-86, 2003.

4. F. Snik and C. U. Keller, "Astronomical Polarimetry: Polarized Views of Stars and Planets,” Planets, Stars and Stellar Systems, pp. 175-221, 2013.

5. D. M. Stam, J. W. Hovenier, and L. B. F. M. Waters, "Using polarimetry to detect and characterize Jupiter-like extrasolar planets,” A\&A, vol. 428, no. 2, pp. 663-672, Dec. 2004.

6. L. V. Ksanfomality, "Search for extrasolar planets by polarimetry," Sol Syst Res, vol. 41, no. 4, pp. 301-306, Aug. 2007. 
7. M. Collados, F. Bettonvil, L. Cavaller, I. Ermolli, B. Gelly, A. Pérez, H. Socas-Navarro, D. Soltau, R. Volkmer, and the EST team, "European Solar Telescope: Progress status," Astron. Nachr., vol. 331, no. 6, pp. 615-619, Jun. 2010.

8. J. Sánchez-Capuchino, M. Collados, D. Soltau, R. López, J. L. Rasilla, and B. Gelly, “Current Concept For The 4-m European Solar Telescope (EST) Optical Design,” International Optical Design Conference and Optical Fabrication and Testing, 2010.

9. S. Keil, T. Rimmele, C. Keller, and The ATST Team, "Design and development of the Advanced Technology Solar Telescope,” Astron. Nachr., vol. 324, no. 4, pp. 303-304, Jun. 2003.

10. S. L. Keil, T. R. Rimmele, J. Wagner, and the ATST team, "Advanced Technology Solar Telescope: A status report,” Astron. Nachr., vol. 331, no. 6, pp. 609-614, Jun. 2010.

11. N. Krishnappa and A. Feller, "Precision in ground-based solar polarimetry: simulating the role of adaptive optics,” Appl. Opt., vol. 51, no. 33, p. 7953, Nov. 2012.

12. R.J. Noll, “Zernike polynomials and atmospheric turbulence”, JOSA, Vol. 66, Issue 3, pp. 207-2011, 1976 